\title{
Egyptian protocol for living donor liver transplantation (LDLT) during SARS-CoV-2 pandemic
}

\author{
Hany Said Abd Elbaset ${ }^{1}$, Ahmad Mohammad Sultan², Iman Fawzy Montasser ${ }^{3}$, Hossam E. M. Soliman \\ Mohamed Elayashy ${ }^{5}$, Nahed A. Makhlouf ${ }^{6^{*}}$ (D) and Scientific Committee of Ministry of Health (MOH) National \\ Project of Waiting Lists, Egypt
}

\begin{abstract}
Background: The current SARS-CoV-2 pandemic may negatively impact the care of liver transplant candidates and recipients.

Main body of the abstract: Accordingly, each country must have its national guidelines based on the current situation and according to available tools. Liver Transplantation Scientific Committee of Waiting List Project in Egypt was established in 13 April 2020. One of the major objectives of this Scientific Committee is the preparation of national protocol for Transplant Centers in Egypt to deal with living donor liver transplantation (LDLT) during SARSCoV-2 pandemic.

Conclusions: The protocol highlights basic hospital requirements for LDLT during SARS-CoV-2 pandemic, the patient selection from the waiting list, management of patients on the waiting list, and post-transplant management.
\end{abstract}

Keywords: SARS-CoV-2, LDLT, Egyptian national protocol, Waiting list project

\section{Background}

COVID-19 is the disease caused by an emerging coronavirus named severe acute respiratory syndrome coronavirus-2 (SARS-CoV-2) that was initially detected in Wuhan City, Hubei Province of China in December 2019. This virus spread across China and then spread worldwide and finally has been declared a pandemic $[1,2]$.

SARS-CoV-2 infection is acquired from anyone who is shedding the virus. Person-to-person transmission was detected through close exposure ( $<6$ feet) to an infected person with COVID-19, mainly via respiratory droplets produced when the infected case coughs or sneezes. The

\footnotetext{
*Correspondence: nahedmak@yahoo.com; nahed.mak@aun.edu.eg Hany Said Abd Elbaset is the Head of the Scientific Committee.

${ }^{6}$ Tropical Medicine and Gastroenterology, Medical Team of Liver Transplantation at Al-Rajhi Liver Hospital, Faculty of Medicine, Assiut University, Assiut, Egypt

Full list of author information is available at the end of the article
}

transmission frequently occurred from symptomatic persons with COVID-19 through droplet spread. Less frequently infection acquired from asymptomatic person has occurred, and the transmission is presumed to be possible from close contact with contaminated objects [1].

Older patients and those with pre-existing medical health problems are at risk of having severe course of disease. It is unclear to what degree chronic liver diseases should be considered as risk factors, due to insufficient clinical studies [3]. However, patients with endstage liver disease are at increased risk of infection due to cirrhosis-associated immune dysfunction [4].

Between 1990 and August 2013, 3804 liver transplants were performed in Arab Countries, with Living Donor Liver Transplantation (LDLT) represented $80 \%$. A lot of Egyptian patients were suffering from end-stage liver disease (ESLD), and necessitating liver transplantation (LT). More than $50 \%$ of transplanted cases in Arab 
countries were reported from Egypt. The regulations for LDLT were made by the Egyptian medical syndicate [5], whereas deceased donor (cadaveric) liver transplant (DDLT) has not yet been authorized. Between July 2018 and January 2020, 380 LDLT operations were performed in Egypt [Data were obtained from Ministry of Health $(\mathrm{MOH})]$ registery database for National Project of Waiting Lists, Egypt).

Information on transplant recipients with COVID-19 are still few and based on published case reports or case series but accumulating experience is going on. However, based on previous data from other viruses including SARS, serious infection in the immunocompromised persons such as organ transplant recipients has happened. Mild affection has also been observed. The risk factors for severe infection have not been completely known. The American Society of Transplantation anticipated a greater viral load and shedding in transplant recipients that could result in more infectivity and spread to other persons [1]. The current pandemic requires unusual allocation of healthcare resources which may negatively impact the care of patients with chronic liver disease [3].

\section{Main text \\ Rational}

Each country with limited resources must have its national guidelines for LDLT during SARS-CoV-2 pandemic based on the current situation and according to the local resources and available tools.

\section{Aims of this protocol}

1- Basic hospital requirements for LDLT during SARS-CoV-2 pandemic.

2 Patient selection from the waiting list.

3 Management of patients on the waiting list for LDLT.

4 Post-transplant management.

\section{Hospital requirements for LDLT during SARS-CoV-2 pandemic}

Basic hospital staff, facilities, and equipment required to restart LDLT program during the pandemic:

a) Presence of sufficient staff for initial evaluation of cases based on symptoms, background, and exposure history (e.g., fever, respiratory symptoms, loss of taste or smell, contact with proven case of SARS-CoV-2 infection) by using rapid questionnaire survey.

b) Nasopharyngeal swab for SARS-CoV-2 PCR laboratory facilities: Nasopharyngeal swab must be done for both recipient and donor $48 \mathrm{~h}$ before hospital admission and $24 \mathrm{~h}$ before the operation.

c) High-resolution CT chest for both recipient and donor on hospital admission.

d) Laboratory facilities and infection control team facilities for screening, early detection of suspected cases including hospital staff, potential donors, and recipients.

e) Provide safe pathway for both recipient and donor including operative rooms, ICU, ward, necessary equipment such as ventilators, ultrasound, and duplex equipment.

\section{Medical staff precautions and protection:}

a) Personal Protective Equipments (PPE) must be provided to all operative team, ICU staff, ward staff, radiology and laboratory staff i.e. (All medical persons and workers who are in contact with donor and recipient in perioperative care) (detailed infection prevention and control procedures during SARS-COV-2 pandemic, Additional file 1: Annex 1)

b) Any medical staff with a history of contact with confirmed cases of COVID-19 without wearing full PPE must be temporary excluded from the team for home isolation for 14 days +/- nasopharyngeal swab if needed (i.e., if any suggestive symptoms appear like fever, cough) [6].

\section{Patient selection from the waiting list}

Basic precautions needed for potential recipients for LDLT:

Recipients of LDLT and donor candidates must have home isolation and personal distancing for a minimum of 10 days, counting back from the planned date of transplantation. Reporting any fever or respiratory symptoms to the transplant team by phone [7].

\section{Initial evaluation before admission:}

Evaluation is based on symptoms, background, and exposure history (e.g., fever, respiratory symptoms, loss of taste or smell, contact with proven case of SARS-COV-2 infection) and by the latest updated rapid survey questionnaire provided and updated by $\mathrm{MOH}$.

\section{Nasopharyngeal swab for SARS-CoV-2 PCR:}

Nasopharyngeal swab must be done for both recipient and donor $48 \mathrm{~h}$ before hospital admission and $24 \mathrm{~h}$ before the operation $[3,7]$.

High-resolution CT chest for both recipient and donor on hospital admission.

As negative nasopharyngeal swab results do not completely exclude SARS-CoV-2 infection [8], so CT chest is mandatory during the pandemic.

The transplant team must explain to potential recipients and their relatives the risk of SARS-CoV-2 
infection aggravation under post-transplant immunosuppression [7].

Recipient selection, Table 1.

"High priority for liver transplantation denotes mortality risk on the waiting list overweighs the risk of SARS-CoV-2 infection.

Moderate risk: Case by case discussion with Liver Transplantation Team, but the Scientific Committee of $\mathrm{MOH}$ national project of waiting lists does not recommend transplantation in the current situation.

Transplant candidate with a history of confirmed COVID-19 disease:

- Non-urgent transplantation should be avoided.

- If urgent transplantation is indicated:

- Nasopharyngeal/oropharyngeal PCR should be negative twice (at least $48 \mathrm{~h}$ apart).

- Complete resolution of clinical, laboratory, or radiological findings.

- Ideal disease-free interval is unknown but not less than 20 days from disease onset, and better to be more than 37 days $[1,9]$.

Recommendations for the donors (pre-operative test and management) $[1,3,10]$ :

a) Only the donor with low risk is accepted.

No direct contact with a positive or suspected patient. No suspicious symptoms as follows:

- Fever $\left(>38^{\circ} \mathrm{C}\right)$.

- Malaise or flu-like symptoms, +/- myalgias.

- New cough.

- Shortness of breath.

- Unexplained abdominal pain, nausea, and/or diarrhea.

- Loss of sense of taste and/or smell. b) Recommendation for home isolation 14 days before hospital admission.

c) Complete blood count (CBC) shows no leucopenia and $\mathrm{C}$-reactive protein (CRP) is negative

d) Radiological chest X-ray and high-resolution CT should be negative for any suspected lesion.

e) Nasopharyngeal/oropharyngeal PCR should be done twice in the week before transplantation with 48-72 $\mathrm{h}$ apart.

f) High-risk donors who have direct contact with positive or suspected patient with any suspicious symptom should be advised to postpone donation for 28 days after symptom resolution and have a negative PCR test.

g) Detailed informed SARS-CoV-2 infection risk consent should be taken from the donor [11]

\section{Management of potential recipients on the waiting list for LDLT}

a) All potential recipients on the waiting list should be regularly followed up either by phone contact or transplant clinic visits whenever needed.

b) Monthly update of Child-Pugh and MELD scores (weekly update if MELD> 25).

c) Abdominal ultrasound/duplex and alpha-fetoprotein every 3 months.

d) Triphasic CT portography if the results of ultrasound/duplex is suspicious.

e) Weekly arrangement of waiting list by liver transplant team in each center according to ChildPugh, MELD score, HCC characteristics of potential recipients.

f) Mortality/dropout on the waiting list must be recorded including date, time on waiting list, and cause of death/dropout.

Table 1 Recipient selection for LDLT during SARS-CoV-2 pandemic

\begin{tabular}{lll}
\hline High priority risk group* & Moderate risk group \\
\hline Criteria & - Fulminant liver failure & $\bullet$ MELD 15-19 \\
& $\bullet$ MELD equal or greater than 20 & $\bullet$ HCC within Milan fit for bridge therapy. \\
& - Child-Pugh class C (score 10). & \\
& - Previous history of HRS. \\
& - Benious history SBP. \\
& - HCC within Milan unfit for bridge (Child-Pugh late B or C). \\
& - HCC beyond Milan within UCSF with a good response of downstaging after \\
& 3 months.
\end{tabular}

Decision Proceed for transplantation with precautions

Complete donor preparation, close follow up on the waiting list

MELD model for end stage liver disease; HRS hepato-renal syndrome; SBP spontaneous bacterial peritonitis; PVT portal vein thrombosis; HCC hepatocellular carcinoma; UCSF University of California San Francisco; ${ }^{*}$ high priority for liver transplantation denotes mortality risk on the waiting list overweighs the risk of SARS-CoV-2 infection 
g) We recommend that specialized centers for liver transplantation provide easily accessible contact information to facilitate immediate hepatology consultations to the local health care providers if needed. h) Include nasopharyngeal swab PCR testing for COVID-19 disease for patients with acute on chronic liver failure [3].

i) Counseling of liver transplant candidates for vaccination against Streptococcus pneumoniae and influenza [3].

j) Detailed informed Covid-19 disease risk consent for recipient before the operation [11]

\section{Post-transplant management}

\section{Immunosuppressant protocol:}

Maintain the usual protocol of immunosuppressants which is based on calcineurin inhibitors (CNIs), mycophenolate mofetil (MMF) and steroid, and at the usually recommended target levels.

For a recipient with proven post-transplant Covid19 disease:

a) Data from different liver transplant centers are scarce and there is no consensus on how to deal with immunosuppressant drugs in such situation. So far there have been no specific recommendations in terms of course or management of liver transplanted patients from major Societies. Some reports suggest decreasing immunosuppression for infected recipients, if no recent rejection episodes. Paradoxically, others suggest that a reactive immune response might be the cause for severe tissue damage and that immunosuppression might be protective from the postulated cytokine storm [12, 13].

b) The national recommendations include reduction/ hold of CNI dose, according to disease severity and radiological finding in $\mathrm{CT}$ chest. This must be done as case by case discussion in a multidisciplinary team with a senior chest consultant, transplant hepatology consultant, and ICU consultant in severe cases.

c) MMF/Azathioprine (AZA) hold should be discussed on a case by case basis. It is essential to consider reducing azathioprine or mycophenolate dosages, especially in the setting of lymphopenia, fever, or worsening pneumonia attributed to COVID-19 [14].

d) Patients who develop adult respiratory distress syndrome (ARDS), use steroid dose as recommended by Covid protocol prepared by $\mathrm{MOH}$ [15]. Methylprednisolone $1 \mathrm{mg} / \mathrm{kg} /$ day for 3-5 days then oral steroids in tapering dose over 4-6 weeks.

e) Drug-drug interactions with immunosuppressant medications need to be evaluated and managed [15]. f) We recommend regular telephone communication between the liver transplant team (or Central Transplant Committee) and health care physicians caring for liver transplant recipient with proven COVID-19 positive at isolation hospital.

\section{Post-transplant clinic and follow up visits:}

a) For recipients who transplanted more than 3 months, the follow-up can be made through telemedicine with the transplant team according to scheduled visits as usual or exceptional telemedicine if any new developed symptoms or whenever needed. Hospital visit and/or admission whenever needed according to clinical judgment by the transplant physician in charge with the case $[12,16]$. All recipients discharged from the hospital with educational booklet information that includes the alarm symptoms (e.g., fever, cough, vomiting, or diarrhea) and contact details for the transplant team members.

b) Early postoperative period: less than 3 months from the operation, regular post-operative follow-up visits as per center protocol. Limit the number of patients who visit the transplant clinic, and limit the number of family members/friends who accompany patients in their visits. Special precautions on hand hygiene, wearing face mask throughout the visit, well-aerated rooms for waiting, keep distance $2 \mathrm{~m}$ between patients at waiting area, and decontamination of the waiting area should be practiced, etc. [12].

c) Transplant recipients should be educated about the importance of performing frequent hand hygiene, cleaning frequently touched surfaces, avoidance of crowded public places, and applying social distancing, staying away from individuals who are ill [17].

\section{Vaccination:}

The usual vaccination of liver transplant recipients against Streptococcus pneumoniae and influenza is strongly recommended [3].

\section{Travel restriction for transplant recipients:}

It is essential to postpone all non-essential travel for transplant recipients. We also recommend that transplant patients' immediate household contacts should not travel to high-risk areas [1]. Recipients transplanted from rural areas are instructed to stay nearby to their transplant centers at least for the first 3 months.

\section{Conclusion}

The preparation of a national protocol for Transplant Centers in Egypt to deal with LDLT during SARS-CoV2 pandemic is essential. This protocol highlighted basic hospital requirements, the patient selection from the waiting list, management of patients on the waiting list, and post-transplant management during SARS-CoV-2 
pandemic. Regular and frequent updates are essentially needed according to any emerging data.

\section{Supplementary Information}

The online version contains supplementary material available at https://doi. org/10.1186/s43066-020-00074-4

Additional file 1: Annex 1. Infection Prevention and Control Procedures in Liver Transplant Centers During SARS-CoV-2 Pandemic.

\section{Abbreviations}

LDLT: Living donor liver transplantation; SARS-CoV-2: Severe acute respiratory syndrome coronavirus-2; COVID-19: Coronavirus disease 2019; ESLD: Endstage liver disease; DDLT: Deceased donor (cadaveric) liver transplant; PPE: Personal protective equipments; MOH: Ministry of health; MELD: Mode for end-stage liver disease; HRS: Hepato-renal syndrome; SBP: Spontaneous bacterial peritonitis; PVT: Portal vein thrombosis; HCC: Hepatocellular carcinoma; UCSF: University of California San Francisco; CNIs: Calcineurin inhibitors; MMF: Mycophenolate Mofetil; AZA: Azathioprine; ARDS: Adult respiratory distress syndrome

\section{Acknowledgements}

The authors would like to thank all members of the national project of management of patients on waiting lists, $\mathrm{MOH}$, Egypt.

\section{Authors' contributions}

HS A and A M S were responsible for the concept, the plan of the work, and final revision. I F M shared in the literature review, design, writing of the manuscript, and initial revision. H E M S and M E shared in writing Additional file 1: Annex 1 and final revision, NA M shared in the literature review, writing of the manuscript, and she is the corresponding author. All Authors have read and approved the final manuscript.

\section{Funding}

Not applicable.

Availability of data and materials

Not applicable.

Ethics approval and consent to participate

Not applicable.

\section{Consent for publication}

Not applicable.

\section{Competing interests}

The authors declare that they have no competing interests.

\section{Author details}

${ }^{1}$ HPB Surgery and Liver Transplantation, Ain Shams University, Cairo, Egypt.

${ }^{2}$ GI Surgery, Liver Transplantation Unit, Gastrointestinal Surgical Center, Mansoura University, Mansoura, Egypt. ${ }^{3}$ Tropical Medicine and Gastroenterology, Ain Shams University, Cairo, Egypt. ${ }^{4}$ Professor of HPB Surgery and Liver Transplantation, National Liver Institute, Menoufia University, Menuofia, Egypt. ${ }^{5}$ Anesthesia, Intensive Care and Pain Management, Cairo University, Cairo, Egypt. ${ }^{6}$ Tropical Medicine and Gastroenterology, Medical Team of Liver Transplantation at Al-Rajhi Liver Hospital, Faculty of Medicine, Assiut University, Assiut, Egypt.

Received: 19 August 2020 Accepted: 27 December 2020 Published online: 09 February 2021

\section{References}

1. American Society of Transplantation (2020) 2019-nCoV (coronavirus): FAQs for organ donation and transplantation https://www.myast.org/sites/default/ files/COVID19\%20FAQ\%20Tx\%20Centers\%2003.20.2020-FINAL.pdf

2. Wang C, Horby Peter W, Hayden Frederick G, Gao George F (2020) A novel coronavirus outbreak of global health concern. Lancet. 395(10223):470-473
3. Boettler T, Newsome PN, Mondelli MU, Maticic M, Cordero E, Cornberg M, Berg T (2020) Care of patients with liver disease during the COVID-19 pandemic: EASL-ESCMID position paper. JHEP Reports 2(3):100113. https:// doi.org/10.1016/j.jhepr.2020.100113

4. Albillos A, Lario M, Álvarez-Mon M (2014) Cirrhosis-associated immune dysfunction: distinctive features and clinical relevance. J Hepatol 61: 1385-1396

5. Amer, Marwan (2016) Living donor liver transplantation in Egypt. Hepato Biliary Surg Nutr 5(2):98-106

6. Egyptian Ministry of Health and Population (2020) Coronavirus disease 2019 (COVID-19) SARS COV2. Management Guide.Vesion 1. Egypt, pp 1-24

7. Japan Society of Transplantation (2020) Basic guidelines in transplantation medicine for new coronavirus infection (COVID-19), 2nd edn

8. Winichakoon P, Chaiwarith R, Liwsrisakun C et al (2020) Negative nasopharyngeal and oropharyngeal swab does not rule out COVID-19. J Clin Microbiol 58:e00297-e00220

9. Zhou F, Yu T, Du R, Fan G, Liu Y, Liu Z et al (2020) Clinical course and risk factors for mortality of adult inpatients with COVID-19 in Wuhan, China: a retrospective cohort study. Lancet 395:1054-1062

10. Fix OK, Hameed B, Fontana RJ, Kwok RM, McGuire BM, Mulligan DC et al (2020) Clinical best practice advice for hepatology and liver transplant providers during the COVID-19 pandemic: AASLD expert panel consensus statement. Hepatology 72(1):287First published: 16 April 2020. https://doi. org/10.1002/hep.31281

11. American Society of Transplantation (2020) COVID-19 organ donation and transplantation town hall (donor issues and candidate concerns): American Society of Transplantation Available from: https://www.youtube.com/ watch?reload $=9 \& v=0 \times$ aFpTba0T8\&t=accessed April 13, 2020

12. AASLD. American Association for the Study of Liver Diseases (2020) Clinical insights for hepatology and liver transplant providers during the COVID-19 pandemic. Released: April 7

13. D'Antiga L (2020) Coronaviruses and immunosuppressed patients. The facts during the third epidemic. Liver Transpl 26(6):832-834

14. Zhu L, Xu X, Ma K, Yang J, Guan H, Chen S et al (2020) Successful recovery of COVID-19 pneumonia in a renal transplant recipient with long-term immunosuppression. Am J Transplant 20(3):1859-1963

15. Egyptian Ministry of Health and Population (2020) Management guidelines for COVID-19. Patients with special medical conditions. Egypt, April

16. Terry K (2020) Telehealth seen as a key tool to fight COVID-19. The hospitalist https://www.thehospitalist.org/hospitalist/article/218574/ coronavirus-updates/telehealth-seen-key-tool-help-fight-covid-19

17. Center for Disease Control and Prevention (CDC) (2019) Coronavirus disease 2019 (COVID-19). Cleaning and disinfection for community facilities https:// www.cdc.gov/coronavirus/2019ncov/community/organizations/cleaningdisinfection.html

\section{Publisher's Note}

Springer Nature remains neutral with regard to jurisdictional claims in published maps and institutional affiliations.

\section{Submit your manuscript to a SpringerOpen ${ }^{\bullet}$ journal and benefit from:}

- Convenient online submission

- Rigorous peer review

- Open access: articles freely available online

- High visibility within the field

- Retaining the copyright to your article

Submit your next manuscript at $>$ springeropen.com 